\title{
PONTOS DE CORTE DO ÍNDICE DE MASSA CORPORAL PARA CLASSIFICAR O ESTADO NUTRICIONAL EM IDOSOS
}

\section{CUT-OFF POINTS OF BODY MASS INDEX TO SORT THE NUTRITIONAL STATUS IN ELDERLY}

\section{PUNTOS DE CORTE DEL ÍNDICE DE MASA DEL CUERPO PARA CLASIFICAR EL ESTADO NUTRICIONAL EN ANCIANOS}

Recebido: 15/06/2014

Aprovado: 02/12/2014

\author{
Talita Inácio Martins ${ }^{1}$ \\ Joilson Meneguci ${ }^{2}$ \\ Renata Damião ${ }^{3}$
}

Os objetivos desta revisão sistemática foram verificar quais são os pontos de corte para a classificação do IMC, qual é o mais utilizado e a sua aplicabilidade em estudos populacionais com idosos. A partir da base de dados BVS, os descritores utilizados para a busca foram índice de massa corporal, estado nutricional e idoso. Foram encontrados 492 artigos, e desses 21 foram excluídos por duplicação, resultando em 471. Apenas 24 atenderam os critérios de inclusão. No total, foram encontradas quatro referências distintas para classificar os pontos de corte do IMC: Lipschitz, World Health Organization, Nutrition Screening Initiative e Organização Pan Americana de Saúde. O ponto de corte do índice de massa corporal mais utilizado pela literatura é a classificação recomendada pela WHO. O IMC é utilizado com o intuito de avaliar o estado nutricional, associar com co-fatores de saúde; e relacionar seus valores e classificação com risco de morbimortalidade.

Descritores: Índice de massa corporal; Estado nutricional; Idoso.

The aim of this systematic review were to assess what are the cutoff points for BMI classification, which is the most used and their applicability in population studies with elderly. From the base of BVS data, the descriptors used for the search were body mass index, nutritional status and elderly. 492 articles were found, and of these 21 were excluded for duplication, resulting in 471 . Only 24 met the inclusion criteria. In total, four distinct references were found to classify the BMI cutoff points: Lipschitz, World Health Organization, Nutrition Screening Initiative and the Pan American Health Organization's cutoff of body mass index most widely used in the literature is recommended classification by WHO. BMI is used in order to assess nutritional status, co-factors associated with health; and relate their values and classification with risk of mortality.

Descriptors: Body Mass Index; Nutrition status; Aged.

Los objetivos de esta revisión sistemática fueron determinar cuáles son los puntos de corte para la clasificación del IMC, que es el más utilizado y su aplicación en estudios de población con edad avanzada. Desde la base de datos BVS, los descriptores utilizados para la búsqueda fueron índice de masa corporal, estado nutricional e anciano. Se encontraron 492 artículos, y de éstos 21 fueron excluidos por la duplicación, lo que resulto en 471. Sólo 24 cumplieron los criterios de inclusión. En total, se encontraron cuatro referencias distintas para clasificar los puntos de corte del IMC: Lipschitz, Organización Mundial de Salud, Nutrition Screening Initiative. El punto de corte del índice de masa corporal más utilizado en la literatura de la Organización Panamericana de la Salud es la clasificación de la OMS. El IMC es utilizado con el fin de evaluar el estado nutricional, cofactores asociados con la salud; y relacionar sus valores y la clasificación con riesgo de morbimortalidad.

Descriptores: Índice de masa corporal; Estado nutricional; Ancianos.

\footnotetext{
${ }^{1}$ Educadora Física. Especialista em Formação Pedagógica para Profissionais de Saúde. Mestre em Educação Física.

2 Educador Físico. Mestre em Educação Física. Doutorando em Atenção à Saúde pela Universidade Federal do Triângulo Mineiro (UFTM).

${ }^{3}$ Nutricionista. Especialista em Nutrição Clínica. Mestre em Epidemiologia. Doutora em Medicina. Pós Doutorado em Saúde Coletiva. Professor Adjunto III no Curso de Graduação em Nutrição e no Mestrado em Educação Física da UFTM.
} 


\section{INTRODUÇÃO}

ados do Instituto Brasileiro de $\mathrm{D}$ Geografia e Estatística demonstram que o Brasil possui 23,5 milhões de pessoas com idade igual ou superior a 60 anos ${ }^{1}$. Com o aumento significativo de idosos em números absolutos como também a média em anos vividos, essa população ganha destaque em estudos epidemiológicos, principalmente na avaliação de fatores determinantes para as doenças crônicas não transmissíveis e seu estado nutricional2 ${ }^{2}$.

Num estudo realizado no Sul do Brasil, identificou-se que as prevalências das doenças que compõem a síndrome metabólica (hipertensão arterial sistêmica, diabetes mellitus, obesidade central e dislipidemia) foram maiores naqueles idosos de ambos os sexos, que apresentaram excesso de peso ${ }^{3}$. Neste sentido, a identificação do estado nutricional dos idosos tem se destacado na literatura devido sua importância e relação de risco com doenças crônicas não transmissíveis ${ }^{2,4,5}$.

Dados da Pesquisa de Orçamento Familiar de 2008/2009 indicaram que, enquanto a prevalência de excesso de peso aumentou a partir dos 60 anos, diminuiu nos grupos etários com idade superior a 75 anos, diagnosticando-se déficit de peso ${ }^{6}$. Manter um estado nutricional adequado no individuo idoso não é uma tarefa fácil, decorrente da presença constante de doenças crônicas não transmissíveis, uso de medicamentos, modificações fisiológicas inerentes à idade que podem interferir no apetite resultando num déficit nutricional, além das condições socioeconômicas. Os problemas nutricionais estão associados ao aumento de morbidades, maiores taxas de mortalidades, suscetibilidade a infecções e impacto negativo na qualidade de vida dos idosos? .

As medidas antropométricas são importantes para avaliar o risco de morbimortalidade e composição corporal, porém poucas são aplicáveis a um grande número de indivíduos. Dentre essas medidas a massa corporal e a estatura são fundamentais para calcular o Índice de Massa Corporal (IMC). Este índice mensura o estado nutricional, além disso, é o mais utilizado por ser simples, de baixo custo, não invasivo, rápida aplicação e de fácil mensuração ${ }^{8}$, principalmente em estudos de base populacional.

Por outro lado, ainda é inexistente na literatura um consenso do ponto de corte do IMC mais adequado para classificar o estado nutricional de idosos. No envelhecimento ocorrem alterações fisiológicas como perda de massa muscular, aumento de adiposidade central $^{9}$, e em média, os idosos apresentam mais gordura corporal do que os adultos jovens ${ }^{10}$.

Inquéritos nacionais como a Pesquisa Nacional sobre Saúde e Nutrição (PNSN) e a Pesquisa de Orçamento Familiar do Brasil (POF 2008/2009) utilizaram o critério recomendado pela Organização Mundial de Saúde (OMS), porém há críticas sobre o uso dos mesmos pontos de corte para classificar desnutrição ou excesso de peso em adultos e idosos, pois mudanças na composição corporal, associadas ao processo de envelhecimento, devem ser analisadas $^{11}$. Com isso, acredita-se que, utilizar pontos de corte que são propostos para adultos para classificar o estado nutricional em idosos, não levaria em consideração as mudanças fisiológicas do envelhecimento.

Neste sentido, os objetivos desta revisão foram verificar quais são os pontos de corte para a classificação do IMC, qual é o mais utilizado e a sua aplicabilidade em estudos populacionais com idosos.

\section{MÉTODO}

Este estudo trata-se de uma revisão sistemática, realizada a partir da seleção 
de artigos originais, sobre o tema: estado nutricional de idosos.

No mês de abril de 2014 foram levantadas e analisadas as publicações em duplicatas relacionadas ao tema de interesse por pesquisadores simultaneamente. A busca foi realizada a partir da base de dados Biblioteca Virtual de Saúde (BVS). Os descritores utilizados foram: "índice de massa corporal", "estado nutricional" e "idoso". Utilizou-se o operador lógico $A N D$ para a combinação dos termos usados durante a busca das publicações.

Os critérios de exclusão foram: (a) estudos que não foram realizados exclusivamente com idosos ( $\geq 60$ anos); (b) artigos publicados a mais de cinco

Figura 1. Organograma dos artigos encontrados. 2014.

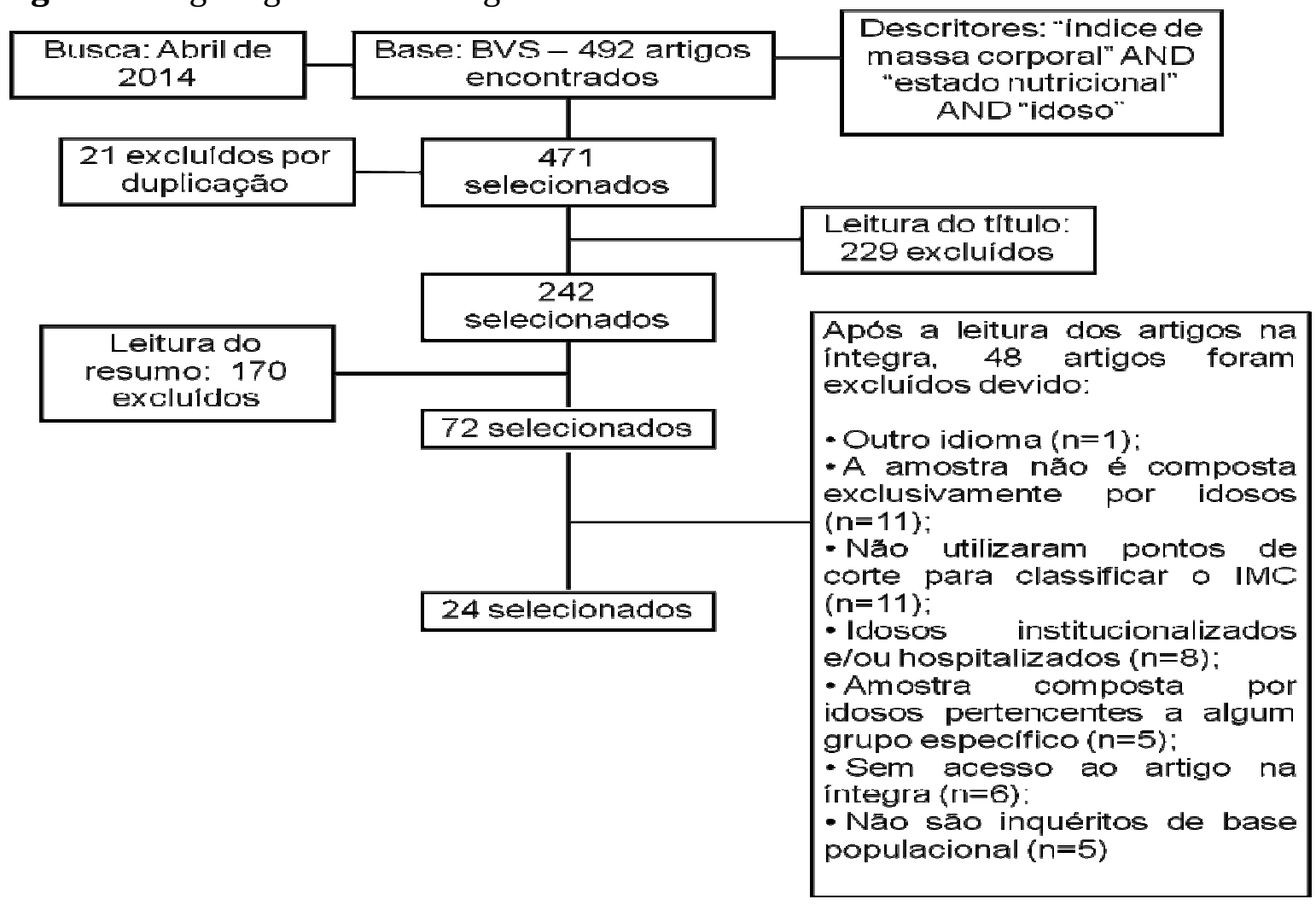

Para a análise dos estudos, foram considerados os seguintes aspectos: ano de publicação; idade e sexo dos participantes; total da amostra; objetivo; desenho; definição operacional para o estado nutricional; pontos de corte do IMC e sua referência; local da coleta dos dados; o que foi analisado a partir dos anos; (c) que não são com inquéritos de base populacional; (d) amostra com idosos institucionalizados ou hospitalizados; (e) amostra composta por idosos pertencentes a algum grupo especifico (exemplo: frágeis, com câncer, limitações físicas); (f) que utilizaram o IMC de forma contínua (g) revisões; (h) artigos que não estejam na língua inglesa, portuguesa e espanhola.

Inicialmente realizou-se uma leitura dos títulos e resumos dos artigos encontrados na busca. Em seguida, os artigos selecionados foram obtidos na íntegra e examinados de acordo com os critérios de exclusão estabelecidos, conforme Figura 1. 
por duplicação, resultando em 471. Posteriormente foram excluídos 229 por leitura do título e 170 do resumo. Após a leitura na integra dos 72 artigos sobre o estado nutricional em idosos, seguindo os critérios de exclusão, apenas 24 foram selecionados (Figura 1). Destes, 37,5\% $(\mathrm{n}=9)$ foram publicados no ano de 2012, $83,3 \% \quad(n=20)$ tinham o desenho transversal, 70,83\% ( $n=17)$ consideraram a faixa etária $\geq 60$ anos, $62,5 \% \quad(n=15)$ foram realizados no Brasil (Quadro 1).

Quadro 1. Características dos estudos sobre o estado nutricional em idosos. 2014.

\begin{tabular}{|c|c|c|c|c|c|}
\hline Autores & Ano & Desenho & Idade & Amostra & Local \\
\hline Andrade et al. & 2009 & Transversal & $\geq 60$ anos & 887 & Vitória/Brasil \\
\hline Silveira et al. & 2009 & Transversal & $\geq 60$ anos & 596 & Pelotas/Brasil \\
\hline Amirkalai et al. & 2010 & Transversal & $\geq 65$ anos & 221 & Tehran/Iran \\
\hline Reyes et al. & 2010 & Transversal & $\geq 60$ anos & 157 & Morelia/México \\
\hline Mastroeni et al. & 2010 & Transversal & $\geq 60$ anos & 218 & Joinville/Brasil \\
\hline Tribess et al. & 2010 & Transversal & $\geq 60$ anos & 265 & Jequié/Brasil \\
\hline Ahn, s. et al. & 2011 & Transversal & $\geq 65$ anos & 1.143 & Estados Unidos \\
\hline Ferreira et al. & 2011 & Transversal & $\geq 60$ anos & 304 & Botucatu/Brasil \\
\hline Freitas et al. & 2011 & Transversal & $\geq 60$ anos & 100 & $\begin{array}{c}\text { Matarazzo e São Miguel } \\
\text { Paulista/Brasil }\end{array}$ \\
\hline Nascto. et al. & 2011 & Transversal & $\geq 60$ anos & 621 & Viçosa/Brasil \\
\hline Sales et al. & 2011 & Longitudinal & 71-81 anos & 1035 & Bambuí/Brasil \\
\hline Silva et al. & 2011 & Transversal & $\geq 60$ anos & 13943 & Brasil \\
\hline Sirola et al. & 2011 & Longitudinal & $\begin{array}{l}\text { Média de } 73 \\
\text { anos }\end{array}$ & 1125 & Finlândia \\
\hline Ahn, s. et al. & 2012 & Longitudinal & $\geq 60$ anos & 3.591 & China \\
\hline Andrade et al. & 2012 & Transversal & $\geq 60$ anos & 833 & Vitória/Brasil \\
\hline Cheserek et al. & 2012 & Transversal & $\geq 60$ anos & 128 & África Oriental \\
\hline Fares et al. & 2012 & Transversal & $\geq 60$ anos & 787 & $\begin{array}{l}\text { Antônio Carlos e } \\
\text { Lafaiete } \\
\text { Coutinho/Brasil }\end{array}$ \\
\hline Lee et al. & 2012 & Transversal & $\geq 65$ anos & 2948 & Tailândia \\
\hline Perera et al. & 2012 & Transversal & $\geq 60$ anos & 437 & $\begin{array}{l}\text { Distrito de Colombo/Sri } \\
\text { Lanka }\end{array}$ \\
\hline Silva et al. & 2012 & Transversal & $\geq 60$ anos & 1441 & Bambuí/Brasil \\
\hline Siqueira et al. & 2012 & Transversal & $\geq 60$ anos & 262 & Bambuí/Brasil \\
\hline Soares et al. & 2012 & Transversal & $\geq 60$ anos & 235 & $\begin{array}{c}\text { Vitória de Santo } \\
\text { Antão/Brasil }\end{array}$ \\
\hline Boscatto et al. & 2013 & Transversal & $\geq 80$ anos & 134 & Antônio Carlos/ Brasil \\
\hline Hsiao et al. & 2013 & Longitudinal & $\geq 75$ anos & 449 & $\begin{array}{c}\text { Pensilvânia/Estados } \\
\text { Unidos }\end{array}$ \\
\hline
\end{tabular}

Observou-se ao todo quatro classificações dos pontos de corte do IMC, sendo eles: Lipschitz ${ }^{12}$, World Health Organization (WHO) ${ }^{13}$, Nutrition Screening Initiative ${ }^{14}$ e Organização Pan Americana de Saúde (OPAS) ${ }^{15}$ (Quadro 2).

A revisão demonstrou que dos 24 artigos $37,5 \%(\mathrm{n}=9)$ utilizaram os pontos de corte do IMC segundo a classificação recomendada pela World Health Organization; 29,2\% (n=7) dos artigos não citam e/ou a referência do ponto de corte utilizado para classificar o IMC é desconhecida (Quadro 2). Em 12,5\% $(n=3)$ utilizam-se duas classificações distintas para comparar e verificar qual o melhor ponto de corte para o IMC $8,16,17$.

0 Quadro 2 demonstra a aplicabilidade do IMC em cada estudo de acordo com o ponto de corte adotado.

Observou-se que o IMC é utilizado com o intuito de avaliar o estado nutricional, principalmente quanto ao baixo e excesso de peso, e associado com co-fatores de saúde; além de relacionar 
seus valores e classificação com risco de morbimortalidade.

Quadro 2. Características dos estudos segundo critérios metodológicos. 2014.

\begin{tabular}{|c|c|c|c|c|}
\hline Autores & Ano & $\begin{array}{l}\text { Ponto de corte e } \\
\text { classificação para o IMC* }\end{array}$ & $\begin{array}{l}\text { Referência } \\
\text { do ponto de } \\
\text { corte do IMC }\end{array}$ & Aplicabilidade do IMC \\
\hline $\begin{array}{l}\text { Andrade } \\
\text { et al. }\end{array}$ & 2009 & $\begin{array}{l}\text { Baixo peso: }<18,5 \\
\text { Normal: } 18,5-24,99 \\
\text { Sobrepeso: } 25-29,99 \\
\text { Obesidade: } \geq 30\end{array}$ & WHO, 1995 & $\begin{array}{l}\text { Determinar } \\
\text { nutricional }\end{array}$ \\
\hline $\begin{array}{l}\text { Silveira et } \\
\text { al. }\end{array}$ & 2009 & 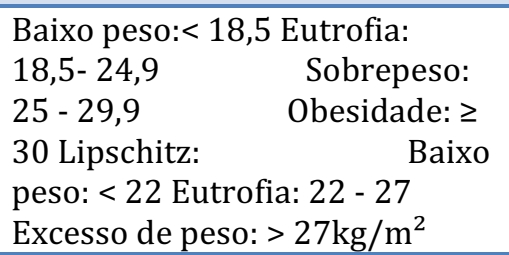 & $\begin{array}{l}\text { WHO, } 1998 \\
\mathrm{e} \\
\text { Lipschitz, } \\
1994\end{array}$ & \begin{tabular}{lcr} 
Comparar & os & \multicolumn{2}{c}{ resultados } \\
(fatores & associados & a \\
obesidade) & por & dois \\
diferentes pontos de & corte \\
do IMC & &
\end{tabular} \\
\hline $\begin{array}{l}\text { Amirkalai } \\
\text { et al. }\end{array}$ & 2010 & $\begin{array}{l}\text { Considerou desnutrição IMC } \\
<24\end{array}$ & $\begin{array}{l}\text { Não } \\
\text { menciona }\end{array}$ & $\begin{array}{l}\text { O IMC é secundário para } \\
\text { comparar com os escores da } \\
\text { mini avaliação nutricional } \\
\text { (MNA) }\end{array}$ \\
\hline $\begin{array}{l}\text { Reyes et } \\
\text { al. }\end{array}$ & 2010 & $\begin{array}{l}\text { Desnutrição: }<20 \\
\text { Peso normal: } 20-25 \\
\text { Excesso de peso: }>25\end{array}$ & $\begin{array}{l}\text { Não } \\
\text { menciona }\end{array}$ & 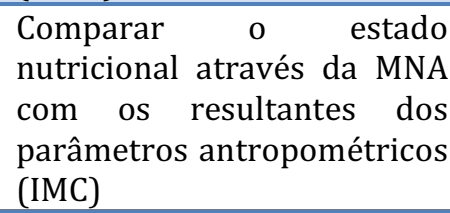 \\
\hline $\begin{array}{l}\text { Mastroeni } \\
\text { et al. }\end{array}$ & 2010 & $\begin{array}{l}\text { Baixo peso: } \leq 23 \\
\text { Peso normal: } 23<\text { IMC }<28 \\
\text { Pré obesidade: } 28 \leq \mathrm{IMC} \leq \\
\text { Obesidade: } \geq 30 \\
\text { Sobrepeso: } \geq 25 \text { (OMS) }\end{array}$ & $\begin{array}{l}\text { OPAS, } 2001 \text { e } \\
\text { WHO, } 2000\end{array}$ & $\begin{array}{l}\text { Verificar o estado nutricional } \\
\text { e comparar a melhor } \\
\text { classificação do IMC e acordo } \\
\text { com seus pontos de corte. }\end{array}$ \\
\hline $\begin{array}{l}\text { Tribess et } \\
\text { al. }\end{array}$ & 2010 & $\begin{array}{l}\text { Peso baixo: }<18,5 \text { Normal: } 18,5 \text { - } \\
24,9 \\
\text { Sobrepeso: } \geq 25\end{array}$ & WHO, 1998 & $\begin{array}{lll}\text { Verificar } & \text { o } & \text { estado } \\
\text { nutricional } & & \end{array}$ \\
\hline Ahn et al. & 2011 & $\begin{array}{l}\text { Peso normal: } 18,5-24,9 \\
\text { Sobrepeso: } 25,0-29,9 \\
\text { Obesidade moderada: } 30-34,9 \\
\text { Obesidade Severa: } \geq 35\end{array}$ & WHO, 1998 & $\begin{array}{l}\text { Determinar a relação entre o } \\
\text { IMC e o estado de saúde. }\end{array}$ \\
\hline $\begin{array}{l}\text { Ferreira } \\
\text { et al. }\end{array}$ & 2011 & $\begin{array}{l}\text { Baixo peso: } \leq 23 \\
\text { Normal: } 23<\mathrm{IMC}<28 \text { Pré- } \\
\text { obesidade: } 28 \leq \mathrm{IMC}<30 \\
\text { Obesidade: } \geq 30\end{array}$ & OPAS, 2001 & $\begin{array}{l}\text { Para classificar os idosos em } \\
\text { obesos e não obesos e } \\
\text { comparar os } \\
\text { associados. }\end{array}$ \\
\hline $\begin{array}{l}\text { Freitas et } \\
\text { al. }\end{array}$ & 2011 & $\begin{array}{l}\text { IMC } \leq 28 \\
\text { Entre } 28 \text { e } 30 \\
\text { Entre } 30-35 \\
\text { IMC }>35\end{array}$ & $\begin{array}{c}\text { Não } \\
\text { menciona }\end{array}$ & $\begin{array}{l}\text { Para classificar e verificar o } \\
\text { estado nutricional. Utiliza } \\
\text { esses pontos de corte, mas } \\
\text { não os classifica. }\end{array}$ \\
\hline $\begin{array}{l}\text { Nascto. et } \\
\text { al. }\end{array}$ & 2011 & $\begin{array}{l}\text { Baixo peso: }<22 \\
\text { Eutrofia: } 22-27 \text { Sobrepeso: }>27\end{array}$ & $\begin{array}{l}\text { Lipschitz, } \\
1994\end{array}$ & Avaliar o estado nutricional \\
\hline $\begin{array}{l}\text { Sales et } \\
\text { al. }\end{array}$ & 2011 & Excesso de peso: $\geq 27$ & $\begin{array}{l}\text { Lipschitz e } \\
\text { Nutritional } \\
\text { Screening } \\
\text { Initiative }\end{array}$ & $\begin{array}{l}\text { Determinar o estado } \\
\text { nutricional, foco no excesso } \\
\text { de peso. }\end{array}$ \\
\hline Silva et al. & 2011 & $\begin{array}{l}\text { Baixo peso: }<18,5 \text { Normal: } \\
\text { 18,50 } \geq \mathrm{IMC} \leq 24,99 \\
\text { Sobrepeso: } 25,00 \geq \mathrm{IMC} \leq 29,99 \\
\text { Obesidade: } \geq 30,00\end{array}$ & WHO, 1995 & $\begin{array}{l}\text { Verificar a prevalência de } \\
\text { excesso de peso (sobrepeso } \\
\text { + obesidade) }\end{array}$ \\
\hline $\begin{array}{l}\text { Sirola et } \\
\text { al. }\end{array}$ & 2011 & Desnutrição: < 21 & $\begin{array}{c}\text { Não } \\
\text { menciona }\end{array}$ & $\begin{array}{l}\text { Utiliza esse ponto de corte } \\
\text { do IMC como sendo um dos }\end{array}$ \\
\hline
\end{tabular}




\begin{tabular}{|c|c|c|c|c|}
\hline & & & & $\begin{array}{l}\text { critérios para o diagnóstico } \\
\text { de fragilidade. }\end{array}$ \\
\hline Ahn et al. & 2012 & $\begin{array}{l}\text { Peso normal: } 18,5-24,9 \\
\text { Sobrepeso: } 25,0-29,9 \\
\text { Obesidade: } \geq 30\end{array}$ & WHO, 1998 & $\begin{array}{l}\text { Analisar fatores associados } \\
\text { ao excesso de peso } \\
\text { (sobrepeso+obesidade) }\end{array}$ \\
\hline $\begin{array}{l}\text { Andrade } \\
\text { et al. }\end{array}$ & 2012 & $\begin{array}{l}\text { Baixo peso: }<18,5 \\
\text { Normal: } 18,5-24,99 \\
\text { Sobrepeso: } 25-29,99 \\
\text { Obesidade: } \geq 30\end{array}$ & WHO, 1995 & $\begin{array}{l}\text { Determinar a quantidade de } \\
\text { idosos classificados com } \\
\text { sobrepeso e obesidade, e a } \\
\text { partir dessa classificação } \\
\text { associar com outras } \\
\text { variáveis. }\end{array}$ \\
\hline $\begin{array}{l}\text { Cheserek } \\
\text { et al. }\end{array}$ & 2012 & $\begin{array}{l}\text { Baixo peso: }<18,5 \\
\text { Normal: } 18,5-24,99 \\
\text { Sobrepeso: } 25-29,99 \\
\text { Obesidade: } \geq 30\end{array}$ & WHO, 1995 & Indicar o estado nutricional \\
\hline $\begin{array}{l}\text { Fares et } \\
\text { al. }\end{array}$ & 2012 & $\begin{array}{l}\text { Baixo peso: }<22,0 \\
\text { Peso adequado: } \\
22,0 \leq \text { IMC } \leq 27,0 \\
\text { Excesso de peso }>27,0\end{array}$ & $\begin{array}{l}\text { Nutrition } \\
\text { Screening } \\
\text { Initiative, } \\
2002\end{array}$ & Verificar o estado nutricional \\
\hline Lee et al. & 2012 & $<17 ; 17-19 ; 19-21 ;>21$ & $\begin{array}{c}\text { Não } \\
\text { menciona** }\end{array}$ & $\begin{array}{l}\text { Verificar as chances de } \\
\text { desnutrição }\end{array}$ \\
\hline $\begin{array}{l}\text { Perera et } \\
\text { al. }\end{array}$ & 2012 & $\begin{array}{l}\text { Baixo peso }<18,5 \\
\text { normal: } 18,5-24,9 \\
\text { Sobrepeso: }>25\end{array}$ & WHO & Avaliar o estado nutricional \\
\hline Silva et al. & 2012 & $\begin{array}{l}\text { Baixo peso: }<18,5 \\
\text { Sobrepeso; } \geq 24,99\end{array}$ & WHO, 1998 & $\begin{array}{l}\text { Verificar o estado nutricional } \\
\text { (desnutrição e sobrepeso) }\end{array}$ \\
\hline $\begin{array}{l}\text { Siqueira } \\
\text { et al. }\end{array}$ & 2012 & Não cita & $\begin{array}{l}\text { Lipschitz, } \\
1994\end{array}$ & $\begin{array}{l}\text { Classificar o estado } \\
\text { nutricional e verificar como } \\
\text { algumas variáveis podem } \\
\text { influenciar no cálculo e } \\
\text { classificação do IMC }\end{array}$ \\
\hline $\begin{array}{l}\text { Soares et } \\
\text { al. }\end{array}$ & 2012 & $\begin{array}{l}\text { Baixo peso: } \leq 22 ; \\
\text { Peso adequado: }>22 \text { e }<27 \\
\text { Sobrepeso: } \geq 27\end{array}$ & $\begin{array}{l}\text { Não } \\
\text { menciona }\end{array}$ & $\begin{array}{lll}\text { Verificar } & \text { o } & \text { estado } \\
\text { nutricional. } & & \end{array}$ \\
\hline $\begin{array}{l}\text { Boscatto } \\
\text { et al. }\end{array}$ & 2013 & $\begin{array}{l}\text { Baixo peso: }<22,0 \\
\text { Excesso de peso: }>25\end{array}$ & $\begin{array}{l}\text { Nutrition } \\
\text { Screening } \\
\text { Initiative, } \\
2002\end{array}$ & $\begin{array}{l}\text { Verificar o estado nutricional } \\
\text { com enfoque na desnutrição } \\
\text { e excesso de peso. }\end{array}$ \\
\hline $\begin{array}{l}\text { Hsiao et } \\
\text { al. }\end{array}$ & 2013 & $\begin{array}{l}\text { Baixo peso: < 18,5 Normal: } 18,5 \\
\text { - 24,9 Sobrepeso: } 25 \text { - } 29,9 \\
\text { Obesidade: } \geq 30 \text { obesidade }\end{array}$ & $\begin{array}{c}\text { Não } \\
\text { menciona }\end{array}$ & $\begin{array}{l}\text { Verificar a prevalência de } \\
\text { obesidade e examinar } \\
\text { associações com fatores de } \\
\text { risco e padrão da dieta. }\end{array}$ \\
\hline
\end{tabular}

*IMC = Índice de massa corporal em $\mathrm{kg} / \mathrm{m}^{2}$

** não menciona a classificação, apenas propõe estes pontos de corte de acordo com a Mini Avaliação Nutricional.

\section{DISCUSSÃO}

Dos artigos analisados a maioria adotaram o ponto de corte da World Health Organization para classificar o IMC18-23, e utilizaram com o intuito de determinar e avaliar o estado nutricional, tanto o excesso de peso quanto a desnutrição, e possíveis fatores associados a estas condições nutricionais. Os demais ${ }^{24-26}$ utilizaram o IMC para classificar o estado nutricional e relaciona-lo com morbidades.

De acordo com a WHO13 a utilização do IMC é indicada para investigar a associação entre o estado nutricional com o risco de morbimortalidade, tanto o excesso quanto o baixo peso traz implicações distintas. As causas de morte associadas com baixo IMC são: tuberculose, doença pulmonar obstrutiva, 
câncer de pulmão e de estômago; e causas associadas com um alto IMC: doenças cerebrovasculares, cardiovasculares, diabetes, e entre os homens câncer no cólon.

Dois artigos adotaram a classificação proposta por Lipschitz ${ }^{27,28}$. Ambos avaliaram o estado nutricional, entretanto Siqueira et $\mathrm{al}^{27}$. além de classificar o estado nutricional dos idosos, verificaram como algumas variáveis podem influenciar no cálculo e classificação do IMC. Outros estudos ${ }^{2,29}$ que adotaram a classificação do Nutrition Screening Initiative também utilizaram com o intuito de verificar o estado nutricional dos idosos. Porém com focos distintos: um na desnutrição e excesso de peso ${ }^{2}$ e o outro para associar com fatores que podem estar relacionados ao estado nutricional desta população ${ }^{29}$. Por outro lado, a classificação da OPAS foi verificada em uma pesquisa ${ }^{30}$ com o objetivo de classificar os idosos em obesos e não obesos e comparar os fatores associados a este estado nutricional que é um dos principais fatores de risco para hipertensão, doença cardiovascular, diabetes tipo 2 e osteoartrite ${ }^{31}$.

Outra investigação ${ }^{17}$ comparou resultados de fatores associados à obesidade por dois pontos de corte distintos do IMC (WHO e Lipschitz) e qual seria o adequado para classificação antropométrica da obesidade, sobre o "olhar" da saúde pública, definições e avanços nas pesquisas, sugeriram que o ponto de corte de obesidade mais sensível para a população idosas brasileira seria o IMC $>27 \mathrm{~kg} / \mathrm{m}^{2}$, ou seja, a classificação de Lipschitz. Em estudo realizado com brasileiros $^{16}$, e adotando este mesmo ponto de corte recomendados por Lipschitz e Nutrition Screening Initiative, com o objetivo de verificar o estado nutricional dos idosos e classifica-los com excesso de peso, destacou que diferenças de coorte de nascimento nas medidas antropométricas de idosos mais velhos, em um futuro próximo, poderão influenciar na maior prevalência de excesso de peso em idosos do sexo masculino.

Mastroeni et al. ${ }^{8}$ com o objetivo de fornecer dados antropométricos de idosos residentes em Joinville, adotaram a classificação da OPAS e da WHO para verificar o estado nutricional dependendo do ponto de corte adotado para o IMC. Verificaram que tanto homens quanto mulheres foram se caracterizaram como eutróficos quando adotaram a classificação da OPAS, em contrapartida com a classificação da WHO que observou-se prevalência de sobrepeso em ambos os sexos.

Dos artigos que não mencionaram a referência dos pontos de corte para classificar o IMC, três utilizam a Mini Avaliação Nutricional (MNA) juntamente com o IMC para diagnosticar o estado nutricional ${ }^{32-34}$. Cervi et al. ${ }^{35}$ sustentam que o IMC não pode ser utilizado como uma única estimativa do estado nutricional dos idosos, pois deve levar em consideração as alterações especificas do envelhecimento, a perda de massa muscular e acúmulo de gordura; além de analisar separadamente as diferentes faixas etárias que compreende a terceira idade.

Três outros estudos mencionaram os pontos de corte para classificar o estado nutricional, porém sem citar a referência respectiva para tal classificação. Tinham como objetivo principal verificar a prevalência de obesidade, examinar associações com fatores de risco e padrão alimentar ${ }^{36-38}$. Apenas uma pesquisa utilizou a classificação do IMC como sendo um dos critérios para diagnosticar a fragilidade ${ }^{39}$.

0 ponto de corte que deve ser adotado para classificar o IMC dependerá do objetivo da pesquisa; ou seja, se o intuito for correlacionar o IMC com a morbimortalidade, o mais recomendado seriam os pontos de corte propostos pela 
Organização Mundial da Saúde e pela Organização Pan Americana de Saúde. Por outro lado, os pontos de corte do Lipschitz e Nutrition Screening Initiative são para classificar o estado nutricional com enfoque principal na desnutrição, que também é comum em pessoas mais velhas e está associada a efeitos adversos significativos á saúde ${ }^{31}$.

Importante ressaltar que valores do IMC estão relacionados tanto a morbimortalidade quanto ao estado nutricional. Esta relação depende do ponto de corte utilizado, levando em consideração a recomendação de cada referência. E, as investigações acabam utilizando para avaliação do estado nutricional, a relação com baixo peso (desnutrição) e com o excesso de peso (risco para obesidade).

\section{CONCLUSÃO}

Pode-se concluir que apesar de não existir um consenso de ponto de corte especifico para os idosos o ponto de corte da Organização Mundial da Saúde foi o mais utilizado nos últimos cinco anos em estudos populacionais para idosos com o objetivo de avaliar somente o estado nutricional e/ou associados a outros cofatores de saúde. Dessa forma, utilizar o mesmo ponto de corte pode auxiliar na comparação dos resultados obtidos nos diferentes estudos epidemiológicos.

Destaca-se como limitação o número considerável de artigos que não apresentaram dados metodológicos completos, restringindo as análises sobre os pontos de corte para classificar o IMC e suas referências em relação aos artigos selecionados que continham todas as informações.

Observou-se que ainda são poucos estudos publicados nos últimos cinco anos relacionados o estado nutricional em idosos. Dentre os pontos fortes da presente revisão destaca-se o cuidado com o levantamento dos dados, cujos estes foram realizados em duplicata para minimizar possíveis falhas de busca, com uso de base de dados confiável.

\section{REFERÊNCIAS}

1. Instituto Brasileiro de Geográfica e Estatística, Características da População e dos Domicílios: Resultados do Universo. IBGE: 2010, 2013. Disponível em: http://www.ibge.gov.br/home/estatistica /populacao/censo2010/caracteristicas d a populacao/caracteristicas da populaca o tab zip xls.shtm).

2. Boscatto C, Duarteb MFS, Coqueiro Rs, Barbosa AR. Nutritional status in the oldest elderly and associated factors. Rev Associação Médica Brasileira. 2013; 59 (Supl.1):40-7.

3. Scherer F, Vieira JLC. Estado nutricional e sua associação com risco cardiovascular e síndrome metabólica em idosos. Rev Nutr. 2010; 23(Supl.3):347-55.

4. Vasconcelos FAG, Cordeiro BA, Rech CR, Petroski EL. Sensitivity and specificity of the body mass index for the diagnosis of overweight/obesity in elderly. Cad. Saúde Pública. 2010; 26(Supl.8):1519-27.

5. Bahat G, Tufan F, Saka B, Akin S, Ozkaya $\mathrm{H}$, Yucel $\mathrm{N}$, et al. Which body mass index (BMI) is better in the elderly for functional status? Archives of Gerontology and Geriatrics. 2012; 54:78-81.

6. Instituto Brasileiro de Geografia e Estatística. Pesquisa de Orçamentos Familiares 2008-2009: Antropométrica e Estado Nutricional de Crianças, Adolescentes e Adultos no Brasil. Rio de Janeiro: IBGE; 2010.

7. Bassler TC, Lei DLM. Diagnóstico e Monitoramento da Situação Nutricional da população idosa no município da região metropolitana de Curitiba (PR). Rev Nutr. 2008; 21(3):311-21.

8.Mastroeni MF, Mastroeni SSBS, Erzinger GS, Marucci, MN. Antropometria de idosos residentes no município de Joinville- SC, Brasil. Rev Brasileira de Geriatria e Gerontologia. 2010; 13(1):29-40. 
9. Bessa LBRS, Barros NV. Impacto da Sarcopenia na Funcionalidade de idosos. Belo Horizonte: UFMG; 2009.

10. Prentice AM, Jebb SA. Beyond body mass index. Obes Rev. 2001;2(3):141-7.

11. Tavares EL, Anjos LA. Perfil antropométrico da população idosa brasileira. Resultados da Pesquisa Nacional sobre Saúde e Nutrição. Cad Saúde Pública. 1999; 15:759-68.

12. Lipschitz DA. Screening for nutritional status in the elderly. Primary Care. 1994; 1(21): 55-67.

13. World Health Organization. Adults 60 year of Age and Older. IN: Phsical Status: The use and Interpretation of Anthropometry. Report of a WHO Expert Committee. WHO 1995; 375-409.

14.Nutrition Screening Initiative. Incorporating nutrition screening and interventions into medical practice: a monograph for physicians. Washington DC: Nutrition Screening Initiative; 1994.

15. Organização Pan-Americana de Saúde. XXXVI Reunión del Comitê Asesor de Investigaciones en Salud - Encuesta Multicêntrica - Salud Beinestar y Envejecimeiento (SABE) en América Latina e el Caribe. Informe preliminar, $2001 . \quad$ Disponível em: http://www.opas.org/program/sabe.htm. 16. Sales ADF, César CC, Lima-Costa MF, Caiaffa WT. Birth cohort differences in anthropometric measures in the older elderly: the Bambuí Cohort Study of Aging (1997 and 2008). Cad Saúde Pública. 2011; 27(3):418-26.

17.Silveira EA, Kac G, Barbosa LS. Prevalência e fatores associados à obesidade em idosos residentes em Pelotas, Rio Grande do Sul, Brasil: classificação da obesidade segundo dois pontos de corte do índice de massa corporal. Cad Saúde Pública 2009; 25(7):1569-77.

18. Andrade FB, Caldas Junior AF, Kitoko PM. Relationship between oral health, nutrient intake and nutritional status in a sample of Brazilian elderly people. Gerodontology. 2009; 26:40-5.

19. Tribess S, Virtuoso Júnior JS, Petroski

EL. Estado nutricional e percepção da imagem corporal de mulheres idosas residentes no nordeste do Brasil. Ciência e Saúde Coletiva. 2010; 15 (1):31-8.

20. Silva VS, Souza I, Petroski EL, Silva DAS. Prevalência e fatores associados ao excesso de peso em idosos brasileiros. Rev Brasileira de Atividade Física e Saúde. 2011; 16 (4):289-94.

21. Cheserek MJ, Waudo JN, Tuitoek PJ, Msuya JM, Kikafunda JK. Nutritional Vulnerability of Older Persons Living in Urban Areas of Lake Victoria Basin in East Africa: A Cross Sectional Survey. J Nutrition in Gerontology and Geriatrics. 2012; 31:86-96.

22. Perera R, Ekanayake L. Relationship between nutritional status and tooth loss in an older population from Sri Lanka. Gerodontology. 2012; 29:566-70.

23. Silva CLA, Lima-Costa MF, Firmo JOA, Peixoto SV. Nível de hemoglobina entre idosos e sua associação com indicadores do estado nutricional e uso de serviços de saúde: Projeto Bambuí. Cad Saúde Pública. 2012; 28(11):2085-94.

24. Ahn S, Sharkey JP, Smith ML, Ory MG, Phillips CD. Variations in Body Mass Index Among Older Americans: The Roles of Social and Lifestyle Factors. J Aging and Health. 2011; 23(2):347-66.

25. Ahn S, Zhao H, Tai-Seale M, Huber JC, Smith ML, Ory MG, et al. The longitudinal effects of behavioral, health, and sociodemographic factors on body mass index among older Chinese adults. International J Public Health. 2012; 5:269-77.

26. Andrade FB, Caldas Junior AF, Kitoko PM, Batista JEM, Andrade TB. Prevalence of overweight and obesity in elderly people from Vitória-ES, Brazil. Ciência e Saúde Coletiva. 2012; 17(3):749-56.

27. Siqueira VO, Costa BVL, Lopes ACS, Santos LC, Lima-Costa MF, Caiaffa WT. Different equations for determining height among the elderly: the Bambuí 
Cohort Study of Aging. Cad Saúde Pública. 2012; 28(1):125-34.

28. Nascimento $\mathrm{CM}$, Ribeiro $\mathrm{AQ}$, Cotta RMM, Acurcio FA, Peixoto SV, Priore SE, et al. Estado nutricional e fatores associados em idosos do Município de Viçosa, Minas Gerais, Brasil. Cad Saúde Pública. 2011; 27(12):2409-18.

29. Fares D, Barbosa AR, Borgatto AF, Coqueiro RS, Fernandes MH. Fatores associados ao estado nutricional de idosos de duas regiões do Brasil. Rev Associação Médica Brasileira. 2012; 58(4):434-41.

30. Ferreira PM, Papini SJ, Corrente JE. Fatores associados à obesidade em idosos cadastrados na rede básica de saúde do município de Botucatu, São Paulo. Rev Ciências Médicas. 2011; 20(4):77-85.

31. Chapman IM. Weight Loss in Older Persons. Med Clin N Am. 2011; 95:579-93. 32. Amirkalai B, Sharifi F, Fakhrzadeh H, Mirarefin M, Ghaderpanahi M, Larijani B. Evaluation of the Mini Nutritional Assessment in the elderly, Tehran, Iran. Public Health Nutrition. 2010; 13(9):1373-9.

33. Reyes MEC, Ramírez FI, Garcia J, Alonso CG, Rodríguez-Orozco AR. Evaluación nutricional comparada del adulto mayor en consultas de medicina familiar. Nutrición Hospitalaria. 2010; 25(4):669-75.

34. Lee L, Tsai AC. Mini-NutritionalAssessment (MNA) without Body Mass Index (BMI) predicts functional disability in elderly Taiwanese. Arch Gerontology Geriatrics. 2012; 54:405-10.

35. Cervi A, Franceschini SCC, Priore SE. Análise crítica do uso do índice de massa corporal para idosos. Rev Nutr. 2005; 18(6):765-75.
36. Freitas AMP, Philippi ST, Ribeiro SML. Listas de alimentos relacionadas ao consumo alimentar de um grupo de idosos: análises e perspectivas. Rev Brasileira Epidemiologia. 2011; 14(1):161-77.

37. Soares LDA, Campos FACS, Araújo MGR, Falcão APST, Lima BRDA, Siqueira DF, et al. Análise do Desempenho Motor associado ao Estado Nutricional de Idosos cadastrados no Programa Saúde da Família, no município de Vitória de Santo Antão-PE. Ciência e Saúde Coletiva. 2012; 17(5):1297-304.

38. Hsiao PY, Mitchell DC, Coffman DL, Wood GC, Hartman TJ, Still C, et al. Dietary Patterns and Relationship to ObesityRelated Health Outcomes and Mortality in adults 75 Years of Age Or Greater. J Nutrition, Health \& Aging. 2013; 17(6):566-72.

39. Sirola J, Pitkala KH, Tilvis RS, Miettinem TA, Strandberg TE. Definition of frailty in older men according to questionnaire data (ranD-36/sf-36): The Helsinki Businessmen Study. J Nutrition, Health \& Aging. 2011; 15(9):783-7.

\section{CONTRIBUIÇÕES}

Talita Inácio Martins foi responsável pelo delineamento do estudo, levantamento dos dados, interpretação e redação do texto. Joilson Meneguci auxiliou no levantamento e analise das publicações em duplicatas. Renata Damião foi responsável pela ideia; foi orientadora da presente revisão e participou da correção da redação do artigo. 